\title{
Quasi-Chemical Reactions in Irradiated Silicon Crystals with Regard to Ultrafast Irradiation
}

\author{
Hrant N. Yeritsyan 1*, Aram A. Sahakyan1, Norair E. Grigoryan1, Vachagan V. Harutyunyan1, \\ Bagrat A. Grigoryan' ${ }^{2}$, Gayane A. Amatuni², Arsham S. Yeremyan ${ }^{2}$, Christopher J. Rhodes ${ }^{3}$ \\ ${ }^{1}$ Department of Applied Physics, Yerevan Physics Institute, Yerevan, Armenia \\ ${ }^{2}$ CANDLE Synchrotron Research Institute, Yerevan, Armenia \\ ${ }^{3}$ Fresh-Lands Environmental Actions, Caversham, UK \\ Email: `grant@yerphi.am
}

How to cite this paper: Yeritsyan, H.N., Sahakyan, A.A., Grigoryan, N.E., Harutyunyan, V.V., Grigoryan, B.A., Amatuni, G.A., Yeremyan, A.S. and Rhodes, C.J. (2018) Quasi-Chemical Reactions in Irradiated Silicon Crystals with Regard to Ultrafast Irradiation. Journal of Modern Physics, 9, 1271-1280.

https://doi.org/10.4236/jmp.2018.96075

Received: April 2, 2018

Accepted: May 21, 2018

Published: May 24, 2018

Copyright (c) 2018 by authors and Scientific Research Publishing Inc. This work is licensed under the Creative Commons Attribution International License (CC BY 4.0).

http://creativecommons.org/licenses/by/4.0/

\section{cc) (i) Open Access}

\begin{abstract}
This paper reports results from an investigation of the interaction of displaced Si-self atoms (I) and their vacancies (V), with impurities in crystalline silicon (Si), as induced by micro-second pulse duration irradiation with electrons at different energies: $3.5,14,25$ and $50 \mathrm{MeV}$ and pico-second pulse duration with energy $3.5 \mathrm{MeV}$. V-V, I-impurity atom and V-impurity atom interactions are analyzed both experimentally and as modeled using computer simulations. A process of divacancy $\left(\mathrm{V}_{2}\right)$ accumulation in the dose-dependent linear region is investigated. The effect of impurities on recombination of correlated divacancies, and I-atoms that had become displaced from regular lattice points is estimated by computer modeling of an appropriate diffusion-controlled process. It is concluded that the experimental results can be interpreted quantitatively in terms of a strongly anisotropic quasi-one-dimensional diffusion of displaced I-atoms. In addition, a significant difference is found between the effects of pico-second duration electron beam irradiation, which causes the formation of A-centre $(\mathrm{V}+$ Oxygen) clusters, while when the beam is applied on a micro-second timescale, divacancies are created instead, although the electrons have the same energy in both cases.
\end{abstract}

\section{Keywords}

Silicon Crystal, Electron Irradiation, Divacancy, Radiation Defects, Introduction Rate, Impurity Atom

\section{Introduction}

The formation of structural defects as a result of applying radiation to silicon crystals $(\mathrm{Si})$ depends both on their impurity content and the lattice homogeneity. 
Although such phenomena have been the subject of numerous previous studies (for example [1]-[6]), there is a lack of detail available regarding the kinetics and accumulation rates for the interactions of impurity atoms with interstitial Si-self atoms (I) and their vacancies (V), i.e. Frenkel pairs, which are induced by the radiation in the Si crystal, in particular, by means of ultrafast radiation-pulses. Information of this kind is of interest from both the fundamental and applied-scientific points of view because it enables conclusions to be drawn regarding the formation of secondary radiation defects. The latter are stable at room temperature), and are the termed the "end-product" of the radiation effect on the Si crystal, which alter the properties of the crystalline material itself, and accordingly those of any devices that are fabricated from it.

Both experimental and theoretical investigations show that, with the exception of GaAs, in general, the effect of the irradiation on the semiconductor crystal, is to induce a high mobility of interstitial atoms and their vacancies (even at temperatures below that of liquid nitrogen), in some cases to the degree that a practically zero-activation energy is observed [7] [8]. As they move, these species undergo reactions with impurities and various crystal imperfections (which exist in the crystal prior to radiation treatment) - such as dislocations and pores, to form new complex radiation defects, which are stable at particular temperatures at which the properties of the crystal are defined. The type of new defect may be a divacancy $\left(\mathrm{V}_{2}\right)$, or complexes that comprise both initially electrically passive (oxygen, carbon) and active impurities (phosphorus, arsenic) in n-Si. The "electrical activation" of passive impurities in Si-samples via irradiation has been demonstrated [4] [9] [10] [11], and the challenge remains to elucidate the nature and distribution of these defects throughout the crystal lattice and to correlate this information with both the energy and dose of the applied radiation, i.e., the so-named "quasi-chemical reaction" which takes place within the irradiated crystal.

In the present work, the interaction of primary radiation defects with the crystal imperfections was not considered, because the samples used were initially almost identical to one another. Furthermore, we may note that the most readily displaced interstitial atoms in $\mathrm{Si}$ are typically impurity atoms from substituted lattice sites, which become mobile and take part at further reactions. In this study, our particular interest is in the formation of divacancies $\left(\mathrm{V}_{2}\right)$, and their rates of introduction and accumulation, since there are a number of different processes by which they can be created during the irradiation process, as "end-product" species, and which depend on the energy of the particular type of radiation being used (in the present work, electrons), which determines the final properties of the silicon crystal.

The main focus of this paper is given to the transfer of energy: from the initial radiation-crystal atom, atom to atom, vacancy-impurity, the formation and accumulation of divacancies, and the rate of production of radiation defects in $\mathrm{Si}$, and the influence of these various factors on the electro-physical and optical properties of the $\mathrm{n}-\mathrm{Si}$ crystals. The experimental measurements involved the 
determination of the concentration and mobility of the free charge carriers, along with infrared (IR) absorption spectroscopy.

\section{Experimental Procedure and Results}

Identical batches of plane-parallel samples, with the (111) crystallographic orientated to a larger face-plane, were fabricated from ingots of $\mathrm{n}$-Si single crystals. Each batch consisted of five types of samples, which differed only in their concentrations of the dominant oxygen $\mathrm{N}_{\mathrm{O}}$ and carbon $\mathrm{N}_{\mathrm{C}}$ impurities:

Type I ( $\mathrm{n}-\mathrm{Si}$ )-Specific resistivity $100 \mathrm{Ohm} \cdot \mathrm{cm}, \mathrm{N}_{\mathrm{O}}=5.0 \times 10^{17} \mathrm{~cm}^{-3}, \mathrm{~N}_{\mathrm{C}}=3.0$ $\times 10^{17} \mathrm{~cm}^{-3}$;

Type II ( $\mathrm{n}-\mathrm{Si})$-Specific resistivity $50 \mathrm{Ohm} \cdot \mathrm{cm}, \mathrm{N}_{\mathrm{O}}=1.0 \times 10^{18} \mathrm{~cm}^{-3}, \mathrm{~N}_{\mathrm{C}}=3.0$ $\times 10^{17} \mathrm{~cm}^{-3}$;

Type III (p-Si)—Specific resistivity $20 \mathrm{Ohm} \cdot \mathrm{cm}, \mathrm{N}_{\mathrm{O}}=1.4 \times 10^{18} \mathrm{~cm}^{-3}, \mathrm{~N}_{\mathrm{C}}=1.5$ $\times 10^{17} \mathrm{~cm}^{-3}$;

Type IV (n-Si)-Specific resistivity $500 \mathrm{Ohm} \cdot \mathrm{cm}, \mathrm{N}_{\mathrm{O}} \leq 10^{16} \mathrm{~cm}^{-3}, \mathrm{~N}_{\mathrm{C}}<10^{16}$ $\mathrm{cm}^{-3}$;

Type V (n-Si)—Specific resistivity $124 \mathrm{Ohm} \cdot \mathrm{cm}, \mathrm{N}_{\mathrm{O}}=5.0 \times 10^{17} \mathrm{~cm}^{-3}, \mathrm{~N}_{\mathrm{C}}=2$ $\times 10^{17} \mathrm{~cm}^{-3}$;

The principal doping impurity concentration did not exceed $10^{14} \mathrm{~cm}^{-3}$.

The experimental geometry was chosen to locate the samples in respect to the electron irradiation beam, such that the maximum possible setting of the irradiation parameters for the whole batch was achieved. The electron irradiation was performed in all, I, II, III, IV types with the following electron beam energies: 3.5, 14, 25 and $50 \mathrm{MeV}$, at the Yerevan Physics Institute, with a beam pulse of about 5 micro-seconds, while type $\mathrm{V}$ samples were irradiated by electrons with an energy of $3.5 \mathrm{MeV}$ at the CANDLE synchrotron Radiation Institute (Armenia) with a beam pulse duration of about one pico-second. Infrared (IR) absorption spectra at $9 \mu \mathrm{m}$ (for impurity oxygen) and at $12 \mu \mathrm{m}$ (for impurity carbon) and Hall-effect measurements were made on the above mentioned samples, both before and after irradiation at room temperature. Details of the Hall-effect measurements [12] and of the IR measurements were as described in works [13] [14]. Note that in the case of 5 micro-seconds beam irradiation a cooling of the samples was applied by liquid nitrogen vapors to the room temperature which do not necessary at pico-second irradiation because the thermal processes do not develop during this very short duration.

The experimental results are indicated in Figures 1-6. Characteristic IR absorption bands due to the localized vibrations from: interstitial oxygen atoms $\mathrm{O}_{\mathrm{I}}$ $\left(1106 \mathrm{~cm}^{-1}\right)$, substitution carbon atoms $\mathrm{C}_{\mathrm{s}}\left(606 \mathrm{~cm}^{-1}\right)$, divacancies $\mathrm{V}_{2}\left(606 \mathrm{~cm}^{-1}\right)$, A-centres $\mathrm{VO}_{\mathrm{I}}\left(829 \mathrm{~cm}^{-1}\right)$ and a complex $\mathrm{VO}_{\mathrm{I}} \mathrm{C}_{\mathrm{I}}\left(866 \mathrm{~cm}^{-1}\right.$, where $\mathrm{C}_{\mathrm{I}}$ is an interstitial carbon atom), are evident in the spectra from irradiated samples of I, II, III types, while in the samples of type IV, only a band related to $V_{2}$ was detected. Hall-effect measurements were carried out to determine the concentration of the main charge carriers, their mobility and formation rate of the radiation defects. 
Figure 1 shows the dose dependences of the concentration of radiation induced divacancies for type I - IV samples, as irradiated by electrons with energies in the range 3.5 to $50 \mathrm{MeV}$.

It is apparent that an additional channel for I in n-Si crystals is introduced at high radiation doses, which begins to compete for the capture of $\mathrm{I}$ on the $\mathrm{C}_{\mathrm{s}}$ sites. Due to the aforementioned higher concentration of $\mathrm{VO}_{\mathrm{I}}$ complexes this channel most likely involves the annihilation of $\mathrm{I}$ and $\mathrm{V}$ on the $\left(\mathrm{VO}_{\mathrm{I}}\right)$ oxygen atoms.

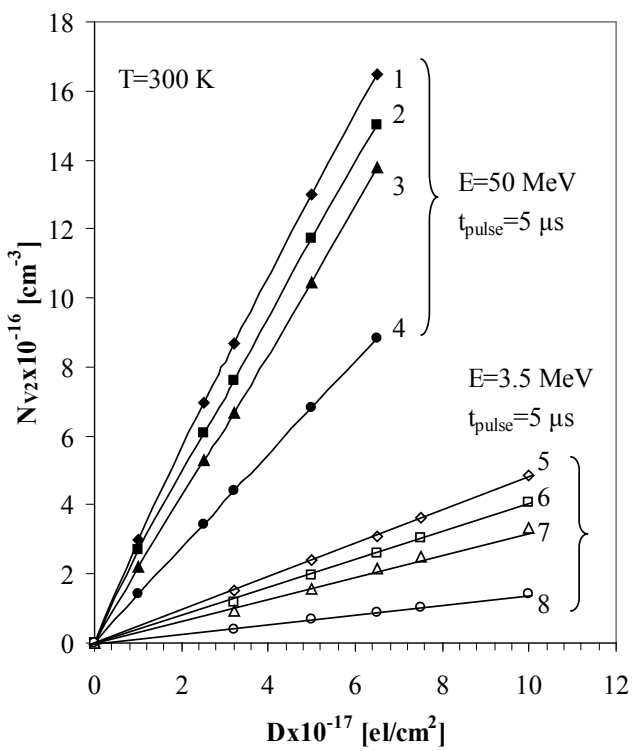

Figure 1. Divacancy concentration $\mathrm{N}_{\mathrm{V} 2}$ as a function of irradiation dose $\mathrm{D}$ in the samples of type I $(1,5)$, II $(2,6)$, III $(3,7)$, I V $(4,8)$ irradiated by $50 \mathrm{MeV}$ $(1,2,3.4)$ and $3.5 \mathrm{MeV}(5,6,7,8$,$) energy electrons.$

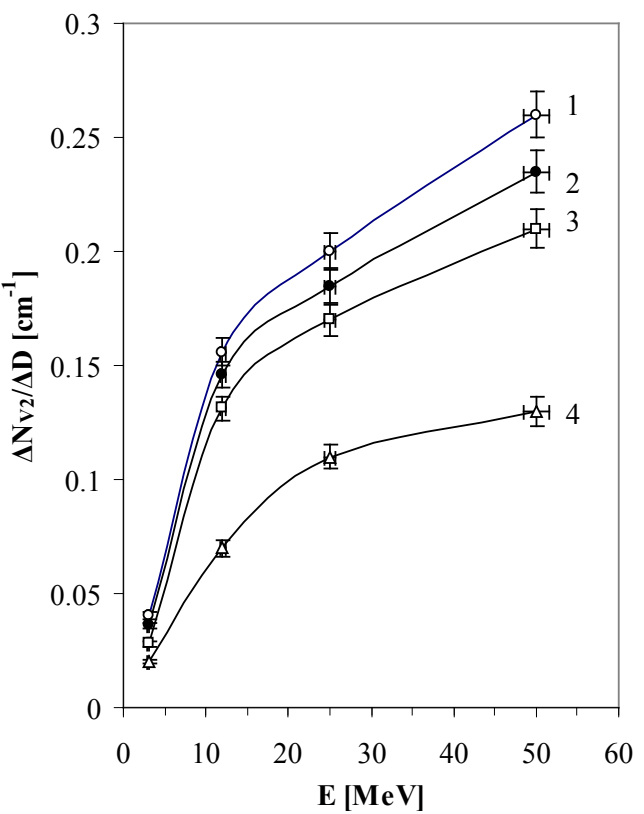

Figure 2. Divacancy introduction rate $\Delta \mathrm{N}_{\mathrm{V} 2} / \Delta \mathrm{D}$ as a function of electron energy $\mathrm{E}$ in the samples of type I (1), II (2), III (3), IV (4). 


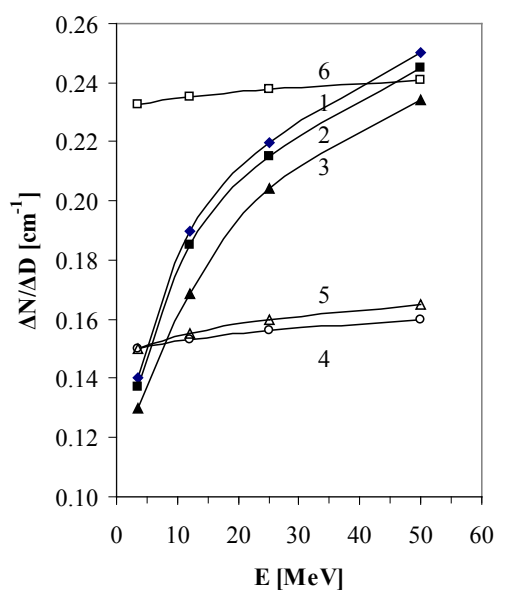

Figure 3. Removal rate $\Delta \mathrm{N} / \Delta \mathrm{D}$ of $\mathrm{C}_{\mathrm{s}}(1,2,3)$ and $\mathrm{O}_{\mathrm{I}}(4,5,6)$ atoms as a function of electron energy $\mathrm{E}$ in the samples type I $(1,4)$, II $(2,5)$ and III $(3,6)$.

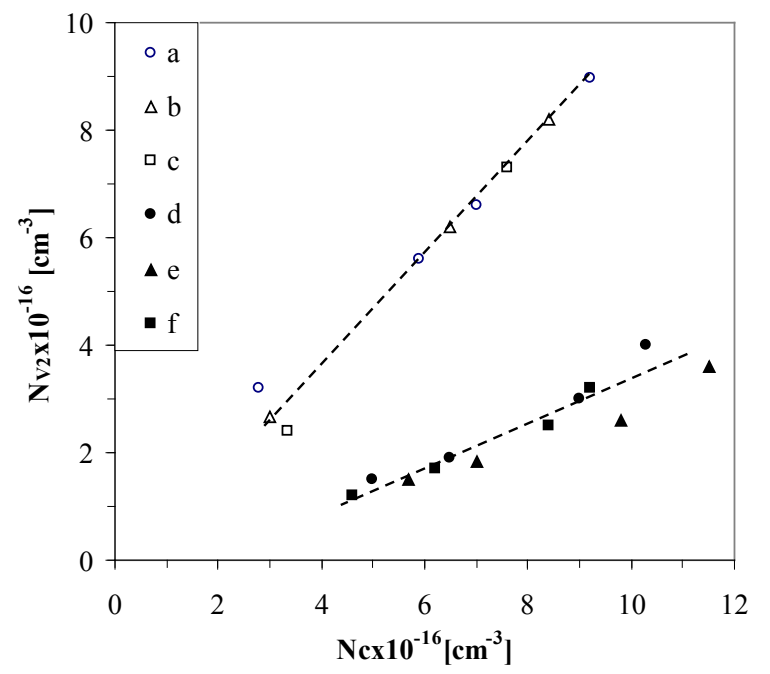

Figure 4. Change in concentration of carbon substitutional atoms $\mathrm{N}_{\mathrm{c}}$ at introduction of $V_{2}$ into the samples type I $(a, d)$, II $(b, e)$ and III $(c, f)$ irradiated by $50 \mathrm{MeV}(\mathrm{a}, \mathrm{b}, \mathrm{c})$ and $3.5 \mathrm{MeV}(\mathrm{d}, \mathrm{e}, \mathrm{f})$ energy electrons.

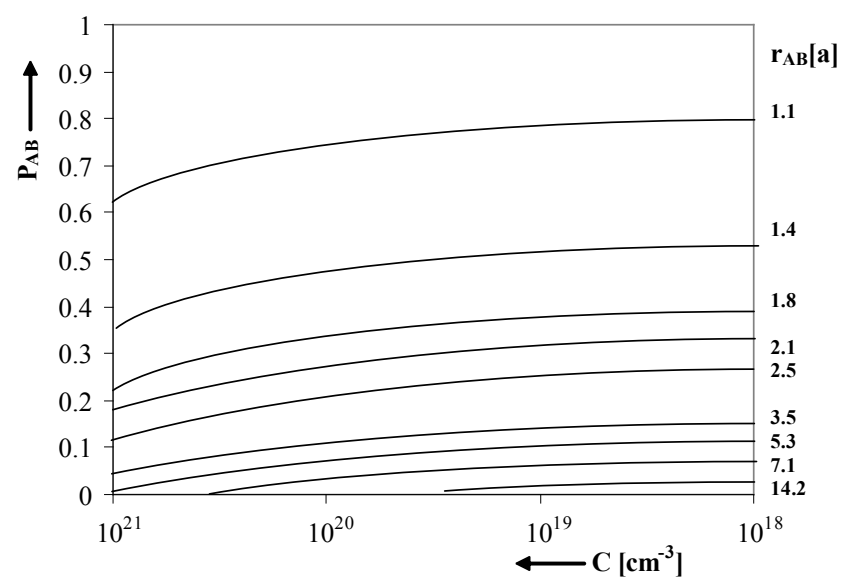

Figure 5. Recombination probability $\mathbf{P}_{\mathrm{AB}}$ as a function of the $\mathrm{C}$ traps concentration at $\mathbf{R}_{B}=1 \mathrm{a}$ and $\mathbf{R}_{C}=0.2 \mathrm{a}$ ( $\mathrm{a}$ is the lattice constant). 


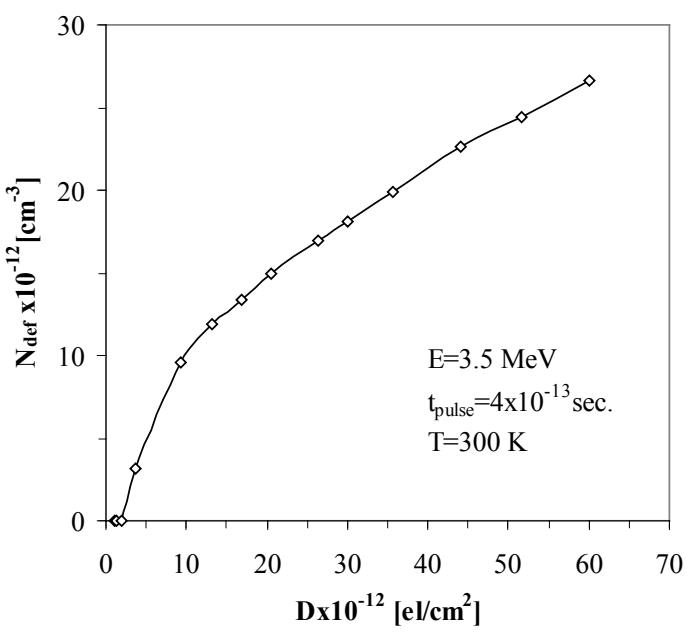

Figure 6. Radiation defects concentration $\mathrm{N}_{\text {def }}$ as a function of irradiation dose $\mathrm{D}$ in the samples of type $\mathrm{V}$ irradiated by $3.5 \mathrm{MeV}$ energy electrons with pulse duration of pico-second region.

In all the samples investigated, the dose dependence of the produced $\mathrm{V}_{2}$ concentration displayed an initial linear region (Figure 1) from the slope of which the introduction rate of $\mathrm{V}_{2}$ at the accumulation stage could be estimated. It is clear from Figure 2 and Figure 3 that the divacancies $\left(V_{2}\right)$ are most effectively introduced into those samples with an enhanced concentration of $\mathrm{C}_{\mathrm{s}}$ atoms, and this effect prevails across the entire electron energy range, as used in this work. It should be noted that the concentration of $\mathrm{C}_{\mathrm{I}} \mathrm{O}_{\mathrm{I}}$ complexes is much lower than the decrease of $\mathrm{C}_{\mathrm{s}}$ concentration and hence, we may conclude that there are more effective sinks for carbon interstitials in the Si crystal, for example, the production of $\mathrm{C}_{s} \mathrm{C}_{\mathrm{I}}$ pairs.

In all cases the formation of $\mathrm{V}_{2}$, the $\mathrm{VO}_{\mathrm{I}}$ (A-centre) and $\mathrm{VO}_{\mathrm{I}} \mathrm{C}_{\mathrm{I}}$ complexes is accompanied by a decrease in the intensities of interstitial oxygen and substitution carbon absorption bands, with the change in $\mathrm{O}_{\mathrm{I}}$ atom concentration proceeding with a nearly constant rate across the electron irradiation energy (from 3.5 to $50 \mathrm{MeV}$ ), while the removal rate of $\mathrm{C}_{\mathrm{s}}$ atom increases with increasing energy (Figure 3). The intensities of the absorbtion bands of Oxygen atoms (O) and Carbon atoms (C) decrease during irradiation because some of these atoms join the mentioned complexes.

For compare results in more detail it is interesting to give numerical calculation concerning radiation defects introduction rate, as an example, for samples with specific resistivity about $100 \mathrm{Ohm} \cdot \mathrm{cm}$ at room temperature: $\Delta \mathrm{N} / \Delta \mathrm{D}=0.049$ $\mathrm{cm}^{-1}$ curve 5 in Figure 1 (micro-second pulse duration $3.5 \mathrm{MeV}$ energy); $\Delta \mathrm{N} / \Delta \mathrm{D}=0.34 \mathrm{~cm}^{-1}$ in Figure 6 (pico-second pulse duration $3.5 \mathrm{MeV}$ energy); $\Delta \mathrm{N} / \Delta \mathrm{D}=0.24 \mathrm{~cm}^{-1}$ curve 1 in Figure 1 (micro-second pulse duration $50 \mathrm{MeV}$ energy); These values confirm our previous results about clusters formation at pico-second pulse duration $3.5 \mathrm{MeV}$ energy irradiation in spite of low energy, low dose and low intensity for this process. We called this phenomenon "Drop dose effect". 


\section{Discussion}

In order to interpret these experimental data, we may consider in detail the generation of radiation defects in Si crystals under irradiation by high energy electrons, and how this varies as a function of electron energy and dose in the particular samples, with specified concentrations of impurities. Following an initial defined stage where simple point defects are introduced in the sample, the probability of $\mathrm{V}-\mathrm{V}$ interactions begins to increase, and the formation of $\mathrm{V}_{2}$ begins. These processes are further influenced by the number of charged impurity species which are created during irradiation as quasi-molecules with initial simple defects (therefore $\mathrm{V}_{2}$ is formed at relatively low irradiation doses in pure $\mathrm{Si}$ ) and low cross-sections for the recombination of species I with those $\mathrm{V}_{2}$. Furthermore, depending on the radiation energy the formation of $V_{2}$ as an initial defect occurs directly via a displaced atom, when this atom, in turn, displaces neighborhood atoms to small lattice constant distances. In this case, the distribution of $\mathrm{V}_{2}$ over the sample is chaotic and the accumulation of these species increases linearly with dose. This is referred to as the formation of a "primary divacancy".

An alternative mechanism is the radiation-induced formation of $\mathrm{V}_{2}$-clusters, when diffusion occurs from the micro-region, where the concentration of primary simple defects (the most mobile interstitials) is high, and secondary $\mathrm{V}_{2}$ are formed in the voids of initially displaced regions, as a consequence of atom-atom cascade collisions. As in the previous case, the accumulation of $\mathrm{V}_{2}$ is linear with dose, but the accumulation rate for this process is higher (Figure 1). This is called a "secondary divacancy". When the initially displaced atom has a low energy, $E_{\mathrm{i}}<\mathrm{E}_{\mathrm{r}}\left(\mathrm{E}_{\mathrm{r}}\right.$-is the threshold of cluster formation, which in $\mathrm{Si}$ is $7.5 \mathrm{keV}$ ), the accumulation of secondary divacancies prevail (Figure 2), while primary vacancies dominate at high energies. A possible explanation of the "impurity effect" at the linear stage of $V_{2}$ accumulation is as follows: the $C_{s}$ atoms are able to efficiently capture the displaced atoms, which inhibits the generated (produced from the same lattice sites) correlated $\mathrm{V}_{2}$ and I atoms from recombining, which promotes an increase in the primary $\mathrm{V}_{2}$ yield. This is supported by the observation of a linear correlation between the increase in $\mathrm{V}_{2}$ concentration and the decreasing concentration of $\mathrm{C}_{\mathrm{s}}$ atoms (Figure 4); capture of I atoms by $\mathrm{C}_{\mathrm{s}}$ atoms will result in their ejection and return to the $C_{I}$ interstitial position [1].

To estimate quantitatively the influence of the capture reaction of the I atoms in regard to their recombination with correlated $\mathrm{V}_{2}$, a computer modeling studies were performed of a pertinent process, according to the following scheme: a three-dimensional isotropic diffusion on a diamond-like lattice of particle $\mathbf{A}$ and subsequent reaction via one of two competing channels-a capture at a spatially fixed centre $\mathbf{B}$ or at randomly volume-distributed traps $\mathbf{C}$. In order to the reaction to proceed, it was necessary for $\mathbf{A}$ to fall into the region of effective spontaneous capture by $B$ or $C$, as defined by the radii $R_{B}$ or $R_{C}$, respectively. Figure 5 presents the probability $\mathbf{P}_{\mathrm{AB}}$ dependence, of a capture at $\mathbf{B}$ at various values of initial distance $\mathbf{r}_{\mathrm{AB}}$ between $\mathbf{A}$ and $\mathbf{B}$. It is clear from Figure 5 that $\mathbf{P}_{\mathrm{AB}}$ 
is insensitive to the $\mathrm{C}$ traps, at concentrations less than $10^{19} \mathrm{~cm}^{-3}$.

The reason for this is that the probability of the particle returning, by executing random walks in an unbounded three-dimensional medium, back to the initial point is less than unity [15] [16] [17], i.e. A will "lose its way" in the infinite volume and will not fall into the $\mathbf{B}$ capture band even in the absence of traps. Hence, given the three-dimensional isotropic diffusion of I atoms, the presence of the impurities capable of capturing them must not affect the linear accumulation of $\mathrm{V}_{2}$.

The situation is different when the diffusion of the displaced I atom is strongly anisotropic. Thus, in the simplest limiting case of one-dimensional motion of the $\mathrm{I}$ atom along a chain connecting $\mathrm{V}_{2}$ and the impurity $\mathrm{C}_{\mathrm{s}}$ atom, a recombination of $\mathrm{V}_{2}$ and the I atom will definitely occur if the latter is not captured by the $\mathrm{C}_{\mathrm{s}}$ atom, and it is the latter that determines the efficiency of the influence of impurities on the primary $\mathrm{V}_{2}$ yield. Thus, a quantitative interpretation of experimental results is possible in a model involving quasi-one-dimensional diffusion of I atoms [12]. In other words the computer modeling simulates the capture process of $\mathrm{A}$ particles by $\mathrm{B}$ and $\mathrm{C}$ traps. The $\mathrm{C}$ type traps are defined by three-dimensional isotropic diffusion of atoms displaced by irradiation, whereas B traps-quasi-one dimensional diffusion. The computer calculation has showed that $\mathrm{C}$ traps don't fit experimental results even depending on irradiation energy (Figures 3-5).

An interesting result is obtained from ultrafast electron irradiation of the samples on the pico-second timescale, as is clear from Figure 6, which shows the dose dependence of the concentration of radiation defects, as induced by electrons with an of energy $3.5 \mathrm{MeV}$. In this case, the dose dependence behavior can been seen to differ from that in Figure 1, which may be explained in terms of the very great difference in the timescales over which the electron beam has been applied. In the case of the ultrafast pulse, a cluster which consists of A-centre (VO) type radiation defects is induced in the silicon crystal, despite the lower irradiation energy for cluster formation [9].

\section{Conclusion}

Results are presented of an investigation of the interaction of displaced Si-self atoms and their vacancies with impurities in crystalline silicon, as a result of irradiation by electrons of different energies, taking into account the influence of the pico-second duration of the electron beam. A process involving the accumulation of radiation defects in the dose-dependent, linear region is studied in detail. The effect of impurities on the recombination of correlated divacancies, with atoms displaced from regular lattice points, is estimated by a computer modeling of an appropriate diffusion-controlled process. It was shown that there is a substantial difference in the introduction rate of radiation defects in Si samples with different contents of impurities, and also when ultrafast electron irradiation is used. The work has particular novelty in that it provides the first analysis of the energy and dose dependences of the introduction and accumulation of 
radiation defects, as a result of electron irradiation in crystalline silicon. It is found that in the case of microsecond pulse duration electron beam irradiation, the induced radiation defects are mainly of the vacancy (initial stage) then divacancy type, while when pico-second pulse duration electron beam (ultrafast) irradiation is used, cluster type associations of A-centers are formed in spite of the same irradiation energy, low dose and low intensity for this process. We called this phenomenon "Drop dose effect".

\section{Acknowledgements}

The authors acknowledge support of this work by the State Committee of Science of the Ministry of Education and Science Republic of Armenia, in the framework of the research project grant No. 17A-1C002.

\section{Conflict of Interests}

The authors declare that they have no conflict of interest.

\section{References}

[1] Bean, A.R., Newman, R.C. and Smith, R.S. (1970) Journal of Physics and Chemistry of Solids, 31, 739-751. https://doi.org/10.1016/0022-3697(70)90207-6

[2] Lang, D.V., Logan, R.A. and Kimerling, L.C. (1977) Physical Review B, 15, 4874-4882. https://doi.org/10.1103/PhysRevB.15.4874

[3] Van Vechten, J.A. (1975) Physical Review B, 12, 1247-1251. https://doi.org/10.1103/PhysRevB.12.1247

[4] Takakura, K., Ohyama, H., Murakawa, H., Yoshida, T., Rafi, J. M., Job, R., Ulyashin, A., Simoen, E. and Claeys, C. (2004) Journal of Applied Physics, 27, 133-136.

[5] Pagava, T.A. and Maisuradze, N.I. (2009) Fizika i Technika Poluprovodnikov, 6, 750-756. (In Russian)

[6] Emtsev, V.V., Ivanov, A.M., Kozlovski, V.V., Lebedev, A.A., Oganesyan, G.A., Strokan, N.B. and Wagner, G. (2012) Fizika i Technika Poluprovodnikov, 46, 473-479.

[7] Khan, A., Yamaguchi, M., Kaneiwa, M., Saga, T., Abe, T., Anzawa, O. and Matsuda S. (2000) Journal of Applied Physics, 87, 8389-8392. https://doi.org/10.1063/1.373552

[8] Vinetski, V.L. and Cholodar, G.A. (1982) Fizika i Technika Poluprovodnikov, 16, 1322-1327.

[9] Yeritsyan, H.N., Sahakyan, A.A., Grigoryan, N.E., Harutyunyan, V.V., Sahakyan, V.A., Khachatryan, A.A., Grigoryan, B.A., Amatuni, G.A., Vardanyan, A.S. and Tsakanov, V.M. (2015) Journal of Modern Physics, 6, p. 2050-2057. https://doi.org/10.4236/jmp.2015.614211

[10] Yeritsyan, H.N., Sahakyan, A.A., Harutyunyan, V.V., Grigoryan, N.E. and Sahakyan, V.A. (2014) Physical Science International Journal, 4, 1225-1234.

[11] Akhmetov, V.D. and Bolotov, V.V. (1979) The Effect of Carbon and Boron on the Accumulation of Vacancy-Oxygen Complexes in Silicon. In: Kekelidze, G.P. and Shakhovtsov, V.I., Eds., Proc. Internat. Conf. Radiation Physics of Semiconductors and Related Materials, Tbilisi, 359-362.

[12] Abgaryan, G.A., Babayan, S.A., Vinetski, V.L., Eritsyan, G.N. and Melkonyan, R.A. 
(1979) Introduction Rate of Divanacies in Silicon by Relativistic Electrons. In: Kekelidze, G.P. and Shakhovtsov, V.I., Eds., Proc. Internat. Conf. Radiation Physics of Semiconductors and Related Materials, Tbilisi, 337-340.

[13] Mukashev, B.N. and Tamendarov, M.F. (1979) Optical Absorption and Electrical Studies of Proton-Bombarded Silicon. In: Kekelidze, G.P. and Shakhovtsov, V.I., Eds., Proc. Internat. Conf. Radiation Physics of Semiconductors and Related Materials, Tbilisi, 341-344.

[14] Watkins, G.D., Troxell, J.R. and Chatterjee, A.P. (1978) Vacancies and Interstitials in Silicon. In: Defects and Radiation Effects in Semiconductors (Inst. Phys. Conf. Ser. 46, London 1979), 16-22.

[15] Kimerling, L.C. (1976) Defect Studies in Electron-Bombarded Silicon. In: Defects and Radiation Effects in Semiconductors (Inst. Phys. Conf. Ser. 31, London, 1977), 221-230.

[16] Montroll, E.W. and Weiss, G.H. (1965) Journal of Mathematical Physics, 6, 167-173. https://doi.org/10.1063/1.1704269

[17] Vinetski, V.L. and Cholodar, G.A. (1979) Radiation Physics of Semiconductors. Naukova Dumka, Kiev, 323 p. 